\title{
Real-Time Control of a Kite-Model using an Auto-Generated Nonlinear MPC Algorithm *
}

\author{
Hans Joachim Ferreau* Boris Houska* Kurt Geebelen** Moritz Diehl ${ }^{*}$ \\ * Eletrical Engineering Department, K.U. Leuven, Kasteelpark \\ Arenberg 10 (bus 2446), 3001 Leuven, Belgium \\ ** Mechanical Engineering Department, K.U. Leuven, Celestijnenlaan \\ 300B, 3001 Leuven, Belgium
}

\begin{abstract}
Airborne wind energy systems like power-generating kites promise to become a sustainable and safe alternative for today's fossil fuel based energy production. Controlling these systems is still a challenge due to the fast and highly nonlinear dynamics. A real-world prototype, which has been build at the K.U. Leuven, consists of a carousel which drives an airplane being attached at one of its arms. In the first part of this paper, we propose a nonlinear grey box model which is developed for controlling this kite carousel. In the second part, we present a code generation tool for nonlinear real-time MPC algorithms which exports plain C-code tailored to particular model dynamics. Numerical closed-loop simulations of the kite carousel show that auto-generated code allows to solve the resulting dynamic optimization problems within less than 900 microseconds.
\end{abstract}

\section{INTRODUCTION}

In the search for a sustainable and safe alternative to today's fossil fuel based energy production, renewable energy sources have received much attention. In particular solar and wind energy is looked upon as a resource large enough to satisfy all of mankind's energy needs. Of these, wind is today by far the cheapest alternative for large-scale production.

The idea of using kites for wind power generation has been previously motivated by Loyd [1980] and recently attracted great attention in the control community, see e.g. Canale et al. [2007, 2010], Houska and Diehl [2007, 2010], Lansdorp et al. [2007], Milanese et al. [2010], Ockels et al. [2006], Williams et al. [2008]. The kite, anchored to a ground-based generator, delivers a high force on the tether while it is being unrolled; this drives the generator to produce electricity. In order to test control systems for kites, a prototype for a kite carousel has been built at the K.U. Leuven. It consists of a rotating device which drives an airplane that is attached at one of its arms as shown in Figure 1. Unrolling the tether allows gaining


Optimization in Engineering (OPTEC) and PFV/10/002, GOA/11/05 Ambiorics, GOA/10/09 MaNet, IOF-SCORES4CHEM, several PhD/postdoc and fellow grants; Flemish Government: FWO: $\mathrm{PhD}$ /postdoc grants, projects: G0226.06, G0321.06, G.0302.07, G.0320.08, G.0558.08, G.0557.08, G.0588.09, research communities (WOG: ICCoS, ANMMM, MLDM), G.0377.09; IWT: PhD Grants, Eureka-Flite+, SBO LeCoPro, SBO Climaqs, SBO POM, O\&ODsquare; Belgian Federal Science Policy Office: IUAP P6/04 (DYSCO); EU: ERNSI, FP7-HD-MPC (INFSO-ICT-223854), COST intelliCIS, FP7-EMBOCON (ICT-248940); Contract Research: AMINAL; Other: Helmholtz-viCERP, ACCM. The first author holds a PhD fellowship of the Research Foundation Flanders (FWO).
}

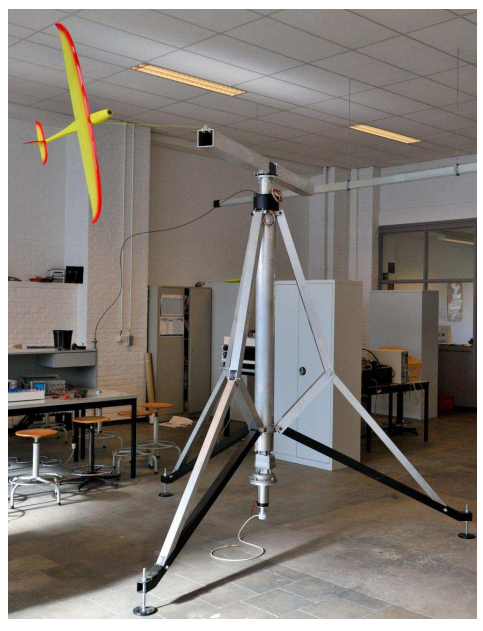

Fig. 1. Experimental setup of the kite carousel.

altitude. This device shall later be used to start and land kites under various wind conditions. As the whole carousel construction has nonlinear dynamics while the airplane (or kite) is moving relatively fast along its orbits, controlling the system reliably is still a challenge.

In order to control systems with nonlinear dynamics, nonlinear model predictive control (NMPC) algorithms are a well-known tool, see Allgöwer and Zheng [2000], Biegler and Rawlings [1991]. Among them, the Continuation/GMRES method by Ohtsuka [2004], the advanced step NMPC controller by Zavala and Biegler [2009] and the real-time iteration (RTI) scheme by Diehl [2001], Diehl et al. [2002] have been proven to be promising approaches for applications with high sampling rates. An overview of existing algorithms for fast nonlinear MPC algorithms can be found in Diehl et al. [2009]. 
The idea to speed-up NMPC calculations by means of code generation has been introduced by Ohtsuka in form of the tool AutoGenU. It exports C-code, and uses a continuation Newton method for the optimality system. At each sampling instant one linear system has to be solved with a GMRES algorithm (see Seguchi and Ohtsuka [2003]). Code generation has also found recent interest in the field of convex optimization (see Mattingley and Boyd [2009]).

The advantages of code generation are at first place the efficiency of the exported plain C-code. In addition, autogenerated code can increase the reliability as all memory can be made static and conditional jumps can be mostly avoided. Finally, plain and self-contained C-code can easily be compiled on embedded hardware. In this paper, we demonstrate that optimized NMPC algorithms based on the RTI scheme - which solves one QP at each iteration allowing for multiple active set changes - can be autogenerated to yield efficient plain C-code. Such a code exported NMPC algorithm is then used to control the nonlinear kite carousel at millisecond sampling times.

We start with developing the nonlinear kite carousel model in Section 2. Section 3 briefly summarizes the real-time iteration scheme, while Section 4 introduces the newly developed software tool for code generation. In Section 5 we demonstrate the performance of the auto-generated code applied to the carousel model. Section 6 concludes.

\section{NONLINEAR KITE MODEL}

In this section, we propose a simplified but nonlinear dynamic model of an airplane which is attached to a carousel. Here, we do not concentrate on high precision models. We rather aim at developing a model which is as simple as possible and still being sufficiently accurate for control.

The arm of the carousel has the length $R$ and rotates with a constant angular velocity $\Omega$ in a horizontal plane. The airplane is connected to the tip of the arm via a tether of constant length $r$. This means that the airplane can move on a sphere centered at the tip of the carousel arm. This is modeled using four differential states that are summarized in the state vector

$$
x:=(\varphi, \vartheta, \dot{\varphi}, \dot{\vartheta})^{T} .
$$

It contains the spherical coordinates $(\phi, \theta)$ of the plane as well as the associated angular velocities $(\dot{\phi}, \dot{\theta})$. Figure 2 shows a sketch of the carousel explaining the choice of the coordinate system.

Note that the equations of motion can be summarized as

$$
\begin{aligned}
& \ddot{\vartheta}=\frac{1}{r}\left[R \dot{\Omega}^{2} \cos (\vartheta) \cos (\varphi)+r(\dot{\Omega}+\dot{\varphi})^{2} \sin (\vartheta) \cos (\vartheta)\right. \\
& \left.\quad+g \sin (\vartheta)+\frac{F_{\vartheta}^{\text {aer }}}{m}\right] \\
& \ddot{\varphi}=\frac{1}{r \sin (\vartheta)}\left[-2 r(\dot{\Omega}+\dot{\varphi}) \dot{\vartheta} \cos (\vartheta)-R \dot{\Omega}^{2} \sin (\phi)+\frac{F_{\varphi}^{\text {aer }}}{m}\right]
\end{aligned}
$$

where $g$ is the gravitational constant and

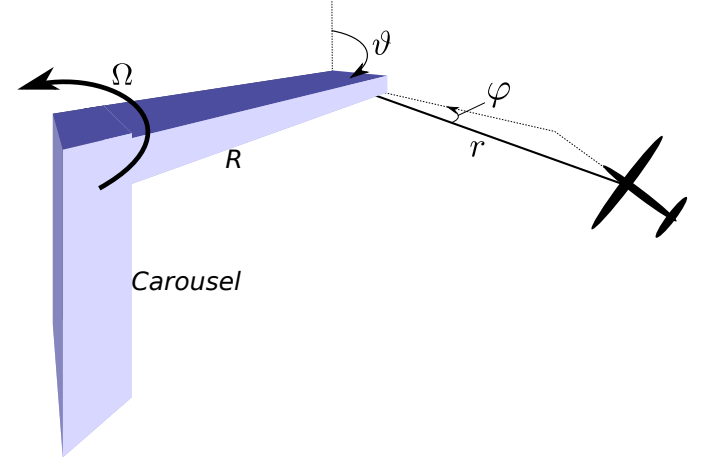

Fig. 2. A sketch of the carousel and the plane together with the position coordinates.

$$
F^{\text {aer }}:=\left(F_{\vartheta}^{\text {aer }}, F_{\varphi}^{\text {aer }}\right)^{T}
$$

summarizes the aerodynamic forces which are defined below.

If there is no external wind, i.e. under laboratory conditions, the effective wind at the plane can in local coordinates be written as

$$
w_{\mathrm{e}}=\left(\begin{array}{c}
\dot{\vartheta} r+R \dot{\Omega} \cos (\vartheta) \sin (\varphi) \\
r(\dot{\Omega}+\dot{\varphi}) \sin (\vartheta)+R \dot{\Omega} \cos (\varphi) \\
R \dot{\Omega} \sin (\vartheta) \sin (\varphi)
\end{array}\right) .
$$

In general the aerodynamic effects at the plane as well as its orientation are quite difficult to model as outlined in Fagiano et al. [2009], Houska and Diehl [2010], Diehl [2002]. However, for controlling the kite it should be enough to use a rough model which contains only the essential physical effects. In this paper, we propose to write the aerodynamic force in the form

$$
F^{\text {aer }}=\frac{\rho A\left\|w_{\mathrm{e}}\right\|^{2}}{2} \psi(x, u)
$$

with $\rho$ being the density of the air, $A$ the wing area, and $\psi: \mathbb{R}^{4} \times \mathbb{R}^{2} \rightarrow \mathbb{R}^{2}$ a function which may in general depend nonlinearly on the states $x \in \mathbb{R}^{4}$ and controls $u \in \mathbb{R}^{2}$. Here, we have made the assumption that the yaw, pitch, and roll rotation of the kite for changing control inputs $u$ is fast compared to the position dynamics in $(\phi, \theta)$ as the function $\psi$ does not have a memory.

Note that many airplane models are based on grey box approaches where the aerodynamic effects at the plane are approximated by employing first or second order Taylor expansions (cf. e.g. Stevens and Lewis [1999]). If the control reactions are small, we may employ this technique as well using an affine or even a quadratic form for $\psi$ whose coefficients must be determined by measurements.

Motivated by the fact that the aerodynamic force $F^{\text {aer }}$ is mainly influenced by the sum of a lift component, which is used to pull the plane in forward direction, and a drag component, which breaks the plane in the opposite direction, we propose the following rough but simple model assumption for the function $\psi$ :

$$
\psi(x, u):=\left(\begin{array}{c}
-C_{\mathrm{L}} u_{1}-b \dot{\vartheta} \\
-C_{\mathrm{D}}\left(1+\alpha_{1} u_{2}\right)-C_{\mathrm{L}}\left(1+\alpha_{2} u_{2}\right) \varphi
\end{array}\right)
$$


Table 1. The parameters for the carousel arm and the attached plane.

\begin{tabular}{|l|l|l|l|}
\hline Parameter & Symbol & Value & Unit \\
\hline length of the arm & $R$ & 1.00 & $\mathrm{~m}$ \\
velocity of the arm & $\Omega$ & 1.00 & $\frac{\mathrm{rad}}{\mathrm{s}}$ \\
mass of the plane & $m$ & 0.80 & $\mathrm{~kg}$ \\
gravitational constant & $g$ & 9.81 & $\frac{\mathrm{m}}{\mathrm{s}^{2}}$ \\
length of the line & $r$ & 1.00 & $\mathrm{~m}$ \\
wing area & $A$ & 0.15 & $\mathrm{~m}^{2}$ \\
density of the air & $\rho$ & 1.20 & $\frac{\mathrm{kg}}{\mathrm{m}^{3}}$ \\
lift coefficient & $C_{\mathrm{L}}$ & 1.00 & - \\
drag coefficient & $C_{\mathrm{D}}$ & 0.15 & - \\
roll stabilization coeff. & $b$ & 15.0 & $\frac{\mathrm{s}}{\mathrm{rad}}$ \\
pitch influence coeff. 1 & $\alpha_{1}$ & 1.00 & - \\
pitch influence coeff. 2 & $\alpha_{2}$ & 0.50 & - \\
\hline
\end{tabular}

with $C_{\mathrm{L}}$ and $C_{\mathrm{D}}$ denoting the nominal lift and drag coefficient, $b$ a roll stabilization coefficient, and $\alpha_{1}$ and $\alpha_{2}$ the relative influence of the pitch control $u_{2}$ on the plane's lift-over-drag efficiency. Note that the other control variable $u_{1}$, is assumed to adjust the roll of the plane. If we have $u_{1}=0$ and $\dot{\vartheta}=0$ - i.e. if we are flying in a horizontal plane at the zero-roll steady state - we expect no further significant aerodynamic forces in $\theta$-direction which motivates the choice of the first component of the coefficient function $\psi$.

The parameters which we are using within this paper are summarized in Table 1. These parameters are estimates which are associated with the real-word experimental setup shown in Figure 1.

In the following, we summarize the above model in form of a nonlinear ordinary differential equation

$$
\dot{x}(t)=f(x(t), u(t)) \quad \text { and } \quad x(0)=x_{0} .
$$

Note that for moderate constant control inputs $u(t)=u_{\text {ref }}$ an associated time constant steady state $x_{\text {ref }}$ exists such that the equilibrium condition $f\left(x_{\text {ref }}, u_{\text {ref }}\right)=0$ is satisfied. For example, for our choice of parameters, we can find an open-loop stable steady state at

$$
\begin{aligned}
& x_{\mathrm{ref}} \approx\left(-0.04 \mathrm{rad}, 1.8 \mathrm{rad}, 0 \frac{\mathrm{rad}}{\mathrm{s}}, 0 \frac{\mathrm{rad}}{\mathrm{s}}\right)^{T} \\
& u_{\mathrm{ref}} \approx(20.5,-0.1)^{T} .
\end{aligned}
$$

The main aim of this paper is to develop and implement an efficient nonlinear real-time algorithm which is capable of controlling the system by tracking the above steady state actively.

\section{THE REAL-TIME ITERATION SCHEME FOR NONLINEAR OPTIMAL CONTROL}

We are interested in solving nonlinear optimal control problems of the form

$$
\begin{aligned}
\min _{x(\cdot), u(\cdot)} & \int_{0}^{T}\left(\left\|x(\tau)-x_{\mathrm{ref}}\right\|_{Q}^{2}+\left\|u(\tau)-u_{\mathrm{ref}}\right\|_{R}^{2}\right) \mathrm{d} \tau \\
& +\left\|x(T)-x_{\mathrm{ref}}\right\|_{S}^{2} \\
\text { s.t. } & \dot{x}(t)=f(x(t), u(t)) \\
& x(0)=x_{0} \\
& \underline{u} \leq u(t) \leq \bar{u} \text { for all } t \in[0, T] .
\end{aligned}
$$

Here, $x: \mathbb{R} \rightarrow \mathbb{R}^{n}$ denotes the state, $u: \mathbb{R} \rightarrow \mathbb{R}^{m}$ the control input, and $\underline{u}, \bar{u} \in \mathbb{R}^{m}$ the control bounds. The right-hand side function $f$ can for example be the kite carousel model from the previous section while the objective is a least-squares tracking term with weighting matrices $Q, S \in \mathbb{R}^{n \times n}$ and $R \in \mathbb{R}^{m \times m}$. Note that optimal control problems of the form (6) occur in the context of nonlinear MPC where $x_{0} \in \mathbb{R}^{n}$ is the current state measurement. The concepts proposed in this paper would also apply to more general optimal control problem formulations. However, we stick to this simplified one as it covers the optimal control problem formulation used for controlling our kite carousel model.

The nonlinear real-time iteration (RTI) scheme has been originally proposed in Diehl et al. [2002]. It is a direct method for optimal control and thus first discretizes the problem (6) and then solves a finite dimensional nonlinear programming (NLP) problem. For the discretization of the nonlinear dynamics a multiple shooting method as proposed by Bock [1983] is employed. The resulting leastsquares NLP problem is solved using a generalized GaussNewton method (see Bock [1983]) which solves one QP at each iteration. This allows for multiple active set changes and thus ensures that the nonlinear MPC algorithm cannot perform worse than a linear MPC controller. Nominal stability of the RTI scheme has been shown in Diehl et al. [2007]. We summarize the main steps of the RTI scheme in Figure 3 and refer to the literature for full details.

Real Time Iterations for Nonlinear MPC:

Initialization: Choose initial values for $x$ and $u$ at all multiple shooting nodes.

\section{Repeat Online:}

1) Evaluate objective function and dynamic system equations and their derivatives at current iterate.

2) Condense resulting large-scale QP into a smaller-scale dense QP.

3) Wait for the measurement $x_{0}$.

4) Compute initial value embedding.

5) Solve dense QP.

6) Send first control immediately to the process.

7) Update current iterate and shift the time.

Fig. 3. Summary of the real-time iteration scheme.

\section{CODE GENERATION}

In order to allow for very fast execution times, we reduce the algorithmic components of the nonlinear real-time iteration scheme to the absolute minimum. Moreover, a symbolic representation of the optimal control problem to be solved is indispensable as this allows for efficient 


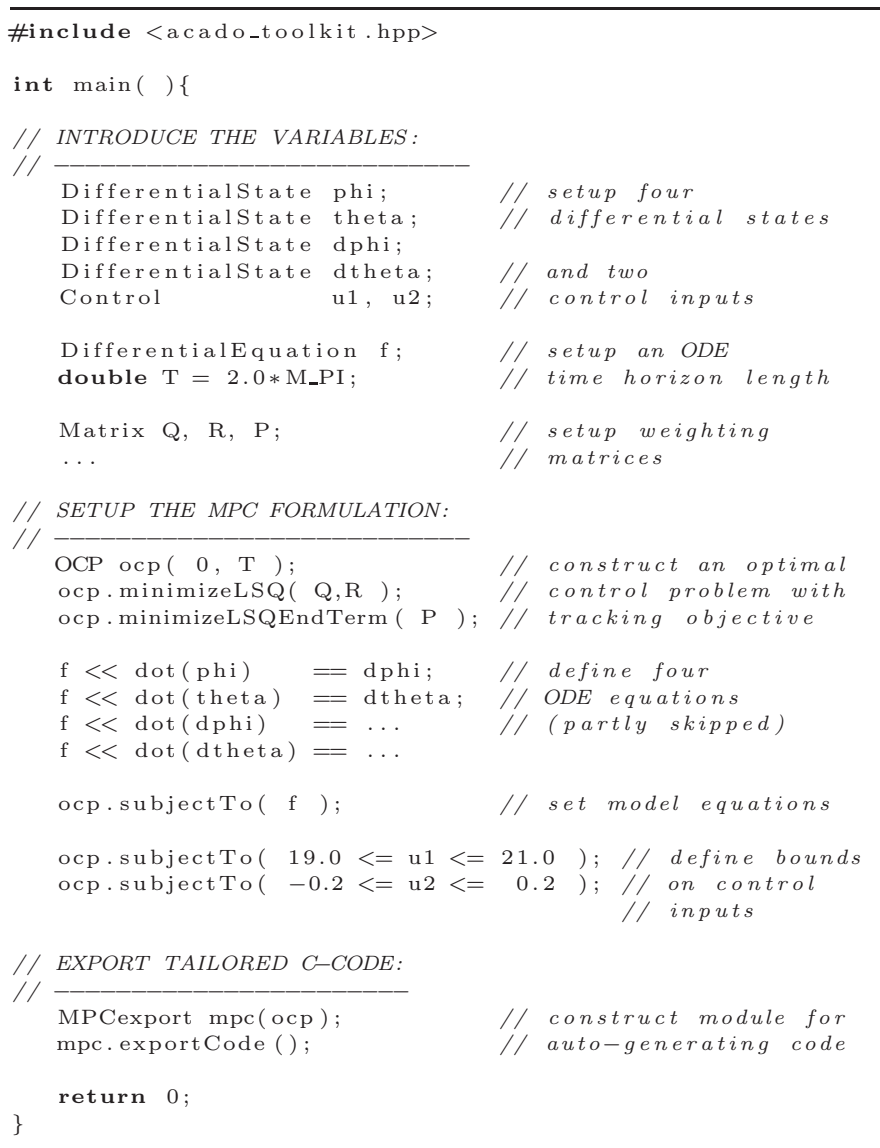

Fig. 4. C++ formulation of a MPC problem for controlling the kite carousel using the symbolic ACADO syntax.

dependency and sparsity detection as well as automatic differentiation to be used for the code export.

The ACADO Toolkit is an open-source software tool which implements algorithms for automatic control and dynamic optimization (see Houska et al. [2011]). It provides user friendly interfaces to setup dynamic optimization and model predictive control problems based on a symbolic $\mathrm{C}++$ syntax. The aim of this section is to explain the newly developed code generation tool, which makes use of the symbolic features of the ACADO Toolkit to export optimized C-code. Here, we follow an idea by Mattingley and Boyd [2009], where automatic generation of C-code for convex optimization was suggested, and extend them to nonlinear dynamic systems.

The ACADO Toolkit allows to define the whole MPC optimization problem based on a symbolic representation, which makes it convenient for a user to setup the problem formulation. Figure 4 shows a code fragment setting up a MPC problem of form (6) to be used for controlling the kite carousel in Section 5. A more detailed documentation and further tutorials on how to specify more general MPC formulations in ACADO can be found on the ACADO Toolkit Homepage [2009-2011].

Once a specific model predictive control problem has been set up with ACADO, we can export the code via the MPCexport class as indicated at the end of the code example in Figure 4. This module will generate optimized C-code which is based on hard-coded dimensions and which uses static memory only. There are four major optimized C-functions generated:

- First, the possibly non-linear right-hand side as well as its derivatives with respect to the states and controls are exported as C-code. Here, the derivatives are symbolically simplified employing automatic differentiation tools and using zero-entries in the Jacobian.

- Second, a tailored Runge-Kutta method for the model equations is generated. Here, we only support the choice of constant step-sizes which can be optimized in offline simulations. This guarantees a deterministic run-time of the simulation. Moreover, the RungeKutta routine also integrates the associated variational differential equations which are needed to compute the sensitivities of the ODE system.

- Third, a discretization algorithm is exported which organizes the single- or multiple-shooting evaluation together with the required linear algebra routines for condensing.

- Fourth, the real-time iteration Gauss-Newton method is auto-generated. At this point, the code generation tool employs a tailored algorithm for solving dense QPs: either the code generation tool CVXGEN Mattingley and Boyd [2009] to export a tailored C-code or an adapted variant of the online QP solver qpOASES (see qpOASES Homepage [2007-2011]) using fixed dimensions and static memory.

Note that the code generation tool generates a code which does not contain any if or switch statements, i.e. we can completely exclude that the program runs into a part of code which we have accidentally never tested. Moreover, there are no malloc/free or new/delete statements in the auto-generated code; all the memory is static and global, while the dimensions are hard-coded. This ensures that we cannot possibly encounter any segmentation faults while running the algorithm on an embedded hardware system. Finally, we avoid for-loops whenever reasonable in order to ensure maximum efficiency.

More details on our code generation ideas for nonlinear MPC can be found in Houska et al. [2011a].

\section{NUMERICAL RESULTS}

In this section we test the closed loop behavior of the autogenerated real-time algorithm. For this aim, we track the steady state reference (5) with a sampling time of $50 \mathrm{~ms}$. In order to setup the online optimal control problem (6), we chose the control bounds

$$
\underline{u}:=(19,-0.2)^{T} \text { and } \bar{u}:=(21,0.2)^{T} .
$$

Moreover, we employ the weighting matrices

$$
Q:=\operatorname{diag}\left(5,1,10 \mathrm{~s}^{2} 10 \mathrm{~s}^{2}\right), \quad R:=\operatorname{diag}(0.005,0.005)
$$

while the matrix $S$ is defined to be the positive definite solution of the algebraic Ricatti equation

$$
A^{T} S+S A-S B R^{-1} B^{T} S+Q=0
$$

with $A:=\frac{\partial f\left(x_{\mathrm{ref}}, u_{\mathrm{ref}}\right)}{\partial x}$ and $B:=\frac{\partial f\left(x_{\mathrm{ref}}, u_{\mathrm{ref}}\right)}{\partial w}$. Finally, the control horizon is set to $T=2 \pi$ seconds. This amounts to the time which the carousel arm needs to complete one full turn. 


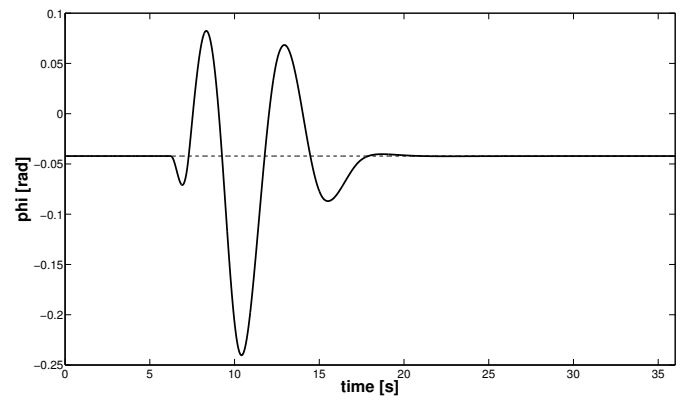

(a) Angular position $\varphi$.

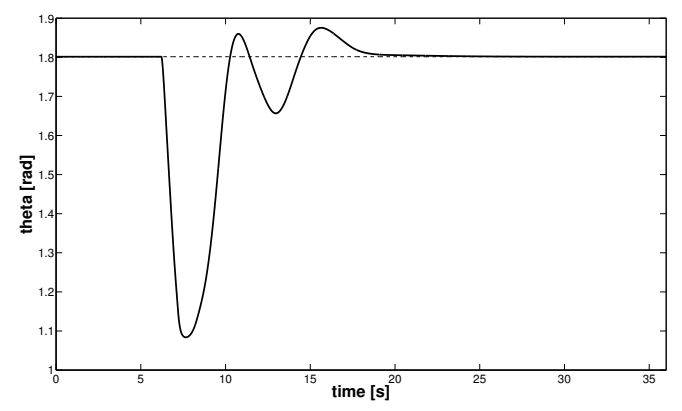

(b) Angular position $\vartheta$.

Fig. 5. Simulated states of the kite carousel (solid lines) together with their respective reference trajectories (dotted lines).

Figure 5 shows a closed loop simulation of the states $\phi$ and $\theta$. During the first $2 \pi \mathrm{s}$ no disturbance occurs. Thus, the controller keeps the kite at its reference position. However, at the time $2 \pi \mathrm{s}$ an external wind gust of $1 \frac{\mathrm{m}}{\mathrm{s}}$ has been simulated. This wind gust takes 1 s until it is switched off again. If the kite would fly open-loop, this disturbance would be large enough to cause a crash. Although the controller has no explicit information about this wind gust, it succeeds in controlling the kite safely back to its reference position. In Figure 6 the corresponding control reaction is shown.

Using a standard PC (Intel Core 2 Duo P9700) with a $2.8 \mathrm{GHz}$ processor and $4 \mathrm{~GB}$ RAM, performing one realtime iteration for the kite carousel formulation took less than $0.9 \mathrm{~ms}$ and was sufficient to track the desired set points. Thus, the auto-generated code could be easily run at the given sampling frequency in real-time even on much slower embedded controller hardware. The major share of the computation was spent within the auto-generated Runge-Kutta integrator of order 4 (using a fixed number of 30 steps per integration) that also generated the required sensitivity information. Table 2 lists the runtimes of all algorithmic sub-tasks for a typical real-time iteration. Note that almost all routines of the auto-generated code have fixed computational load. The only exception is the employed active-set QP solver whose runtime depends on the number of active set-changes and was varying between 10 and 80 microseconds in our simulation.

Note that the computation times obtained by employing the auto-generated NMPC algorithm are orders of magnitude faster than the ones reported by Ilzhoefer et al.

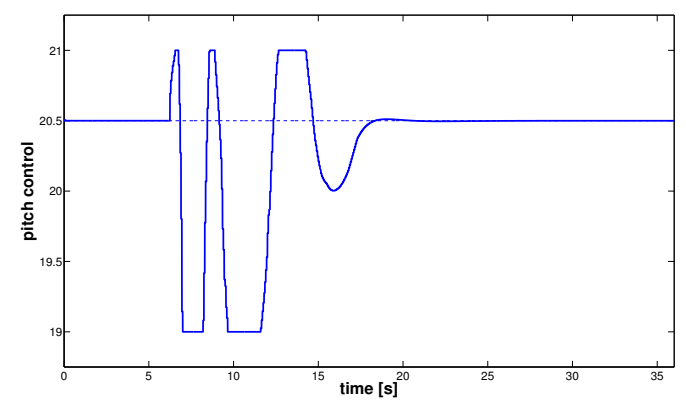

(a) Pitch control $u_{1}$.

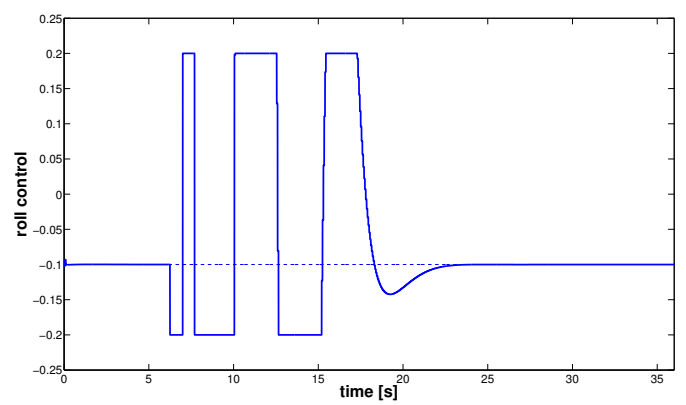

(b) Roll control $u_{2}$.

Fig. 6. Simulated control inputs of the kite carousel (solid lines) together with their respective reference trajectories (dotted lines).

\begin{tabular}{|l|c|c|}
\hline & CPU time & Percentage \\
\hline Integration \& sensitivities & $711 \mu \mathrm{s}$ & $84 \%$ \\
Condensing & $71 \mu \mathrm{s}$ & $8 \%$ \\
QP solution (with qpOASES) & $34 \mu \mathrm{s}$ & $4 \%$ \\
Remaining operations & $27 \mu \mathrm{s}$ & $<4 \%$ \\
\hline A complete real-time iteration & $843 \mu \mathrm{s}$ & $100 \%$ \\
\hline
\end{tabular}

Table 2. Run-time of the auto-generated NMPC algorithm applied to the kite carousel.

[2007]. Therein, one real-time iteration took about $300 \mathrm{~ms}$ using an only slightly more complicated ODE model.

\section{CONCLUSION}

In this paper, we aimed at controlling a kite carousel using nonlinear MPC at millisecond sampling times. To this end, we derived a four states ODE model capturing the main dynamics of the carousel that is suited for optimal control. Moreover, we presented a novel automatic code generation technique for nonlinear MPC algorithms. After summarizing the nonlinear real-time iteration scheme, which has originally been developed in Diehl et al. [2002] for large scale chemical processes, we explained how it can be tuned to achieve ultra-fast computation times for embedded applications. The presented code generator exports optimized C-code for model simulation, sensitivity generation and the Gauss-Newton algorithm, which is based on a single- or multiple shooting discretization of the nonlinear control problem. 
When applying the auto-generated code to the kite carousel setup, we obtained computation times of less than $900 \mu$ s per real-time iteration allowing to run the NMPC algorithm in real-time even on slow embedded controller hardware. Actually using the auto-generated code on an experimental kite carousel setup will be a subject of future research. Another important topic for future research will be to employ a sparse QP solver that can directly deal with the un-condensed, large-scale quadratic program. This would speed-up the solution of MPC problems with long control horizons.

\section{REFERENCES}

ACADO Toolkit Webpage. http://www.acadotoolkit.org, 2009-2011.

qpOASES Webpage. http://www.qpOASES.org, 20072011.

F. Allgöwer and A. Zheng. Nonlinear Predictive Control, volume 26 of Progress in Systems Theory. Birkhäuser, Basel Boston Berlin, 2000.

L.T. Biegler and J.B Rawlings. Optimization approaches to nonlinear model predictive control. In W.H. Ray and Y. Arkun, editors, Proc. 4th International Conference on Chemical Process Control - CPC IV, pages 543-571. AIChE, CACHE, 1991.

H.G. Bock. Recent advances in parameter identification techniques for ODE. In P. Deuflhard and E. Hairer, editors, Numerical Treatment of Inverse Problems in Differential and Integral Equations. Birkhäuser, Boston, 1983.

M. Canale, L. Fagiano, and M. Milanese. Power kites for wind energy generation - fast predictive control of tethered airfoils. IEEE Control Systems Magazine, pages 25-38, 2007.

M. Canale, L. Fagiano, and M. Milanese. High Altitude Wind Energy Generation Using Controlled Power Kites. IEEE Transactons On Control Systems Technology, 18: $168-180,2010$.

M. Diehl. Real-Time Optimization for Large Scale Nonlinear Processes. PhD thesis, Universität Heidelberg, 2001. http://www.ub.uni-heidelberg.de/archiv/1659/.

M. Diehl. Real-Time Optimization for Large Scale Nonlinear Processes, volume 920 of Fortschr.-Ber. VDI Reihe 8, Meß-, Steuerungs- und Regelungstechnik. VDI Verlag, Düsseldorf, 2002. Download also at: http://www.ub.uniheidelberg.de/archiv/1659/.

M. Diehl, H.G. Bock, J.P. Schlöder, R. Findeisen, Z. Nagy, and F. Allgöwer. Real-time optimization and Nonlinear Model Predictive Control of Processes governed by differential-algebraic equations. J. Proc. Contr., 12(4): 577-585, 2002.

M. Diehl, R. Findeisen, and F. Allgöwer. A Stabilizing Real-time Implementation of Nonlinear Model Predictive Control. In L. Biegler, O. Ghattas, M. Heinkenschloss, D. Keyes, and B. van Bloemen Waanders, editors, Real-Time and Online PDE-Constrained Optimization, pages 23-52. SIAM, 2007.

M. Diehl, H. J. Ferreau, and N. Haverbeke. Nonlinear model predictive control, volume 384 of Lecture Notes in Control and Information Sciences, chapter Efficient
Numerical Methods for Nonlinear MPC and Moving Horizon Estimation, pages 391-417. Springer, 2009.

L. Fagiano, M. Canale, M. Milanese, and P. Laface. Control of Tethered Airfoils for High-Altitude Wind Energy Generation. PhD thesis, Politecnico di Torino, 2009.

B. Houska and M. Diehl. Optimal Control for Power Generating Kites. In Proc. 9th European Control Conference, pages 3560-3567, Kos, Greece, 2007.

B. Houska and M. Diehl. Robustness and Stability Optimization of Power Generating Kite Systems in a Periodic Pumping Mode. In Proceedings of the IEEE MultiConference on Systems and Control, Yokohama, Japan, 2010.

B. Houska, H.J. Ferreau, and M. Diehl. ACADO Toolkit An Open Source Framework for Automatic Control and Dynamic Optimization. Optimal Control Applications and Methods, (DOI: 10.1002/oca.939), 2011 (in print).

B. Houska, H.J. Ferreau, and M. Diehl. An auto-generated real-time iteration algorithm for nonlinear MPC in the microsecond range. Automatica, 2011 (submitted).

A. Ilzhoefer, B. Houska, and M. Diehl. Nonlinear MPC of kites under varying wind conditions for a new class of large scale wind power generators. International Journal of Robust and Nonlinear Control, 17(17):15901599, 2007.

B. Lansdorp, R. Ruiterkamp, and W.J. Ockels. Towards Flight Testing of Remotely Controlled Surfkites for Wind Energy Generation. In AIAA Modelling and Simulation Technologies Conference, Hilton Head, South Carolina, USA, 20-23 August 2007.

M.L. Loyd. Crosswind Kite Power. Journal of Energy, 4 (3):106-111, July 1980.

J. Mattingley and S. Boyd. Automatic Code Generation for Real-Time Convex Optimization. Convex Optimization in Signal Processing and Communications, Y. Eldar and D. Palomar, Eds., Cambridge University Press, 2009.

M. Milanese, L. Fagiano, and D. Piga. Control as a key technology for a radical innovation in wind energy generation. Proc. of American Control Conference, 2010.

W.J. Ockels, R. Ruiterkamp, and B. Lansdorp. Ship propulsion by Kites combining energy production by Laddermill principle and direct kite propulsion. In Kite Sailing Symposium, Seattle, USA, 2006.

T. Ohtsuka. A Continuation/GMRES Method for Fast Computation of Nonlinear Receding Horizon Control. Automatica, 40(4):563-574, 2004.

H. Seguchi and T. Ohtsuka. Nonlinear Receding Horizon Control of an Underactuated Hovercraft. International Journal of Robust and Nonlinear Control, 13(3-4):381398, 2003.

B.R. Stevens and F.L. Lewis. Aircraft Control and Simulation. Wiley and Sons, 1999.

P. Williams, B. Lansdorp, and W. Ockels. Optimal Crosswind Towing and Power Generation with Tethered Kites. Journal of Guidance, Control, and Dynamics, 31 (1):81-92, January-February 2008.

V. M. Zavala and L.T. Biegler. The Advanced Step NMPC Controller: Optimality, Stability and Robustness. Automatica, 45:86-93, 2009. 